\title{
An Analysis of Three Dimensional Radiation Patterns from Intrinsic Josephson Junctions with Hot Spot
}

\author{
H. Asai \\ Electronics and Photonics Research Institute, National Institute of Advanced Industrial Science and Technology (AIST), Tsukuba, Ibaraki \\ 305-8568, Japan \\ M. Tachiki, and K. Kadowaki \\ Faculty of Pure and Applied Science, University of Tsukuba, Tsukuba, Ibaraki 305-8573, Japan
}

\begin{abstract}
In this study, we investigate numerically the terahertz radiation from the mesa-structured intrinsic Josephson junctions (IJJs). The Sine-Gordon equation for inside of the mesa, and the Maxwell equation for outside of the mesa, are solved simultaneously. We consider the influence of a hot spot in the mesa where $j_{c}$ locally decreases, and calculate the radiation power as a function of the voltage. We observe strong radiation when the ac Josephson frequency satisfies the cavity resonance condition. For this strong radiation from the IJJs mesa, we calculate the radiation patterns. The radiation patterns reflect the existence of two types of internal modes: a uniform background mode and a cavity resonance mode. Furthermore, the radiation patterns are strongly affected by the diffraction at substrate edges.
\end{abstract}

\section{Introduction}

High- $T_{c}$ superconductors have received much attention as strong candidates for compact solid-state $\mathrm{THz}$ source after Ozyuzer et al., reported radiation of coherent $\mathrm{THz}$ wave from $\mathrm{Bi}_{2} \mathrm{Sr}_{2} \mathrm{CaCu}_{2} \mathrm{O}_{8+\delta}$ (Bi2212) single crystal [1]. The Bi2212 forms intrinsic Josephson junctions (IJJs), that is, natural stacks of Josephson junctions composed of the stacking of superconducting $\mathrm{CuO}_{2}$ layers and insulating layers. Thus, the ac Josephson currents flow through the crystals under dc bias voltages. Intense electromagnetic (EM) radiation has been reported in both experimental and theoretical studies when the ac Josephson frequencies satisfy the cavity resonant frequencies which are determined by the mesa geometies [1-17]. However, the precise nature of the radiation mechanism is not fully understood.

The radiation pattern is a one of a fundamental property of a EM wave emitter for practical use. Furthermore the radiation patterns give us a clues to elucidate the internal EM modes. Klemm and Kadowaki have recently investigated the internal mode of the IJJs by analyzing the experimental radiation patterns [2-4] using antenna theory [18-20]. For the interpretation of the experimental results, they assumed the existence of two types of radiation sources: the uniform part of $a c$ Josephson current and the non-uniform part of ac Josephson current corresponding to cavity resonant modes. However, they did not calculate the phase dynamics in the IJJs. On the other hand, Matsumoto and Koyama numerically investigated the phase dynamics in the IJJs explicitly and calculated the radiation pattern using a two-dimensional model [13-15]. They reported strong emissions from IJJs in the cavity resonance conditions and discussed the relation between the internal modes and the radiation patterns. However, in two-dimensional model, we can not treat correctly the EM field emitted from three-dimensional rectangular IJJs. Moreover, the radiation patterns emitted by inhomogeneous samples have not been investigated in previous studies. Recently, Wang et al., have reported the appearance of hot spots where the temperature is locally high[21-23], and these inhomogeneities are considered to affect the emission from the IJJs.

Email address: hd-asai@aist.go.jp (H. Asai) 


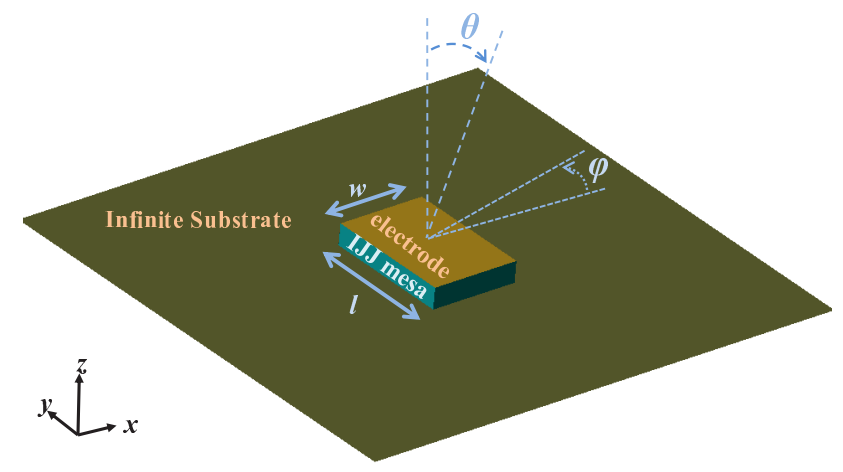

Figure 1: (color online) A schematic view of the three-dimensional calculation model. $w$ and $l$ are the width and the length of the mesa.

In this paper, we present a three-dimensional simulation of the radiation from IJJs mesa to clarify the relation between EM modes in the mesa and the radiation patterns. In particular, we focus on the radiation from the IJJS having a hot spot. We calculate the radiation power as a function of the voltage and observe strong radiation when the ac Josephson frequency satisfies the cavity resonance condition. For this strong radiation from the IJJs mesa, we calculate the radiation patterns. The radiation patterns reflect the existence of two types of internal modes, that is, a uniform background mode and a cavity resonance mode. Furthermore, we find that the radiation patterns are strongly affected by the diffraction at substrate edges.

\section{Calculation Method}

In Fig. 1, we show the schematic figure of three dimensional calculation model. We consider a rectangular IJJs mesa whose IJJs stack along the $z$ axis. The mesa is sandwiched by an substrate and an upper electrode whose geometry is the same as the mesa. We treat the substrate and the electrode as perfect electric conductors. The uniform external current parallel to the $z$ axis is injected from the upper electrode. We assume that the substrate have infinite size in the $x-y$ plane. The dimensions of the mesas are as follows: width $w=0.48 \lambda_{c}$, length $l=0.72 \lambda_{c}$ and thickness $h=0.02 \lambda_{c}$, where $\lambda_{c}$ is the magnetic penetration depth along the IJJ plane.

In this study, we use the finite-difference time-domain method for calculating the EM field inside and outside of the mesa. In the regions outside the IJJs, we solve the three-dimensional Maxwell equations. Inside the IJJs, we solve the dynamical equations of the phase difference and the EM field which are described as follows [13, 14, 17],

$$
\begin{array}{r}
\frac{\partial}{\partial t^{\prime}} P=E_{z}^{\prime}, B_{x}^{\prime}=-\frac{\partial}{\partial y^{\prime}} P, B_{y}^{\prime}=\frac{\partial}{\partial x^{\prime}} P, \\
\frac{\partial}{\partial t^{\prime}} E_{z}^{\prime}=\frac{1}{\epsilon_{c}}\left(\frac{\partial}{\partial x^{\prime}} B_{y}^{\prime}-\frac{\partial}{\partial y^{\prime}} B_{x}^{\prime}\right)+j_{e x t}^{\prime}-j_{c}^{\prime}(x, y) \sin P-\beta E_{z}^{\prime},
\end{array}
$$

where, $P$ is the phase difference between the IJJs, $E_{z}^{\prime}$ is the electric field, $B_{x}^{\prime}$ and $B_{y}^{\prime}$ are oscillation parts of magnetic field, $j_{c}^{\prime}$ is the critical current, and $j_{\text {ext }}^{\prime}$ is the homogeneous external current injected into the IJJs. The parameter $\beta=4 \pi \lambda_{c} \sigma_{c} /\left(c \sqrt{\epsilon_{c}}\right)$ is the normalized conductivity and $\epsilon_{c}$ is the dielectric constant of the junctions along the $c$ axis. In this study, we take $\epsilon_{c}=16$ and $\beta=0.075$. Here, we assume the in-phase motion of the phase differences. In the above equations, the dimensionless quantities are used: length $x^{\prime}=x / \sqrt{\epsilon_{c}} \lambda_{c}$, time $t^{\prime}=\omega_{p} t$ where $\omega_{p}=c / \sqrt{\epsilon_{c}} \lambda_{c}$, EM field $E^{\prime}=\left(2 e d / \hbar \omega_{p}\right) E, B^{\prime}=\left(2 e d / \hbar \omega_{p}\right) B$ where $d$ is the thickness of the insulating layers of IJJs, and the current $j^{\prime}=\left(8 \pi^{2} d \lambda_{c}^{2} / c \phi\right) j$. The far field radiation patterns are calculated from the equivalent electric and magnetic current along the surface of the calculation region[24] .

For investigating the radiation from the inhomogeneous mesa, we consider the appearance of "hot spot" where the temperature is locally high. In our model, such hot spot is simulated by decreasing the local critical current. We take $j_{c}^{\prime}(x, y)=1-\gamma$ in the hot spot region $\left(x_{s}<x<x_{e}, y_{s}<y<y_{e}\right)$ and $j_{c}^{\prime}(x, y)=1$ in the other region. The center of the mesa is located at the origin, and we choose $x_{s}=-0.24 \lambda_{c}, x_{e}=-0.08 \lambda_{c}, y_{s}=-0.36 \lambda_{c}, y_{e}=-0.12 \lambda_{c}$. Figure 2 shows 


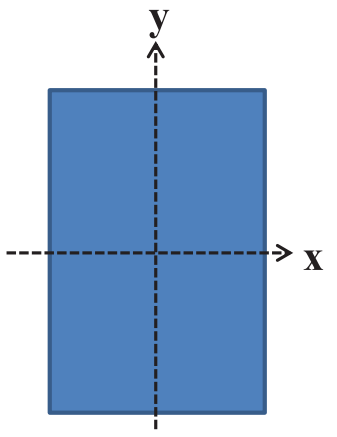

Homogeneous mesa

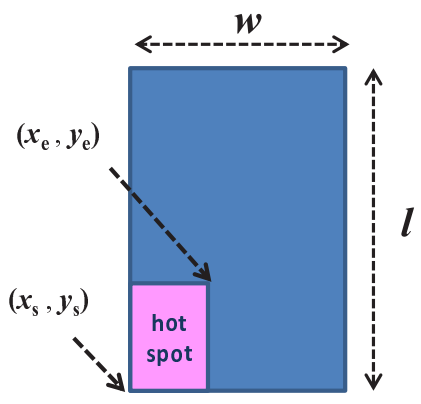

Inhomogeneous mesa

Figure 2: (color online) Schematic figures of the homogeneous mesa (left) and the inhomogeneous mesa (right). $\left(x_{s}, y_{s}\right)$ and $\left(x_{e}, y_{e}\right)$ are the coordinates of the two opposite corners of the hotspot.

schematic figures of the geometries of the mesas. Tthe radiation from the homogeneous mesa are also investigated as a reference. The radiation for various hot spot positions were discussed in our previous work [17].

\section{Results and discussions}

First, we examine the radiation power versus voltage $(P-V)$ curves of the mesas. In Figs. 3 (a) and (b), we show the $P$ - $V$ curves of the homogeneous mesa and the inhomogeneous mesas respectively. Here, the power and the voltage are normalized by $P_{0}=c \Phi_{0}^{2} / 16 \pi d^{3}$ and $V_{p}=\hbar \omega_{p} / 2 e$ respectively. For the inhomogeneous mesa, we calculate the $P-V$ curves for $\gamma=0.2$ and 0.3. In these figures, we can see sharp peaks at voltages where the ac Josephson frequency $f_{\mathbf{J}}=2 e V / h$ satisfies the cavity resonance condition $f_{\mathbf{J}}=c \sqrt{(m / 2 w)^{2}+(n / 2 l)^{2}} / \sqrt{\epsilon_{c}}$, where $m$ and $n$ are arbitrary integers. The peak voltage $V=6.44 V_{p}$, for example, satisfies $(m, n)=(1,0)$. The labels of the peaks in Figs. 3 indicate the indices of the cavity resonance modes. As can be seen from these figures, the cavity resonant peaks

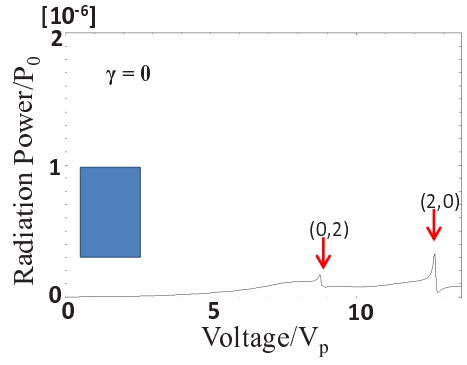

(a)

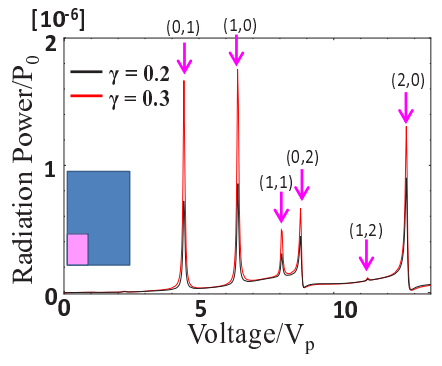

(b)

Figure 3: (color online) (a) The radiation power versus voltage $(P-V)$ curves of the homogeneous mesa. (b) The $P-V$ curves of the inhomogeneous mesa having hot spots. The black and red lines indicate the curves for $\gamma=0.2$ and $\gamma=0.3$ respectively.

whose $m$ and $n$ are even appear in the both mesas. Meanwhile, the peaks whose $m$ and $n$ are odd only appear in the inhomogeneous mesa. In the mesa-structured IJJs, the excitation of the EM mode comes from the ac Josephson current flowing through the mesa, and the distribution of the $a c$ Josephson current has the symmetry corresponding to the mesa structure unless the solitonic states appear. Hence, in a homogeneous rectangular mesa, the odd-numbered cavity modes whose electric fields are asymmetric with respect to the center of the mesa cannot be excited. On the other hand, in the inhomogeneous mesa, the odd-numbered cavity modes appear because the hot spots break the 


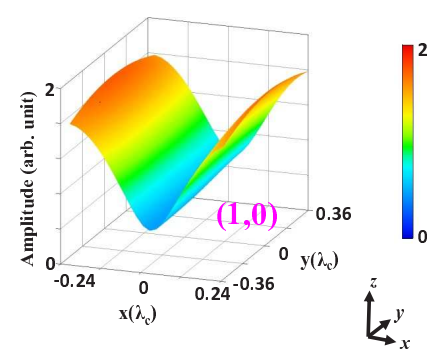

(a)

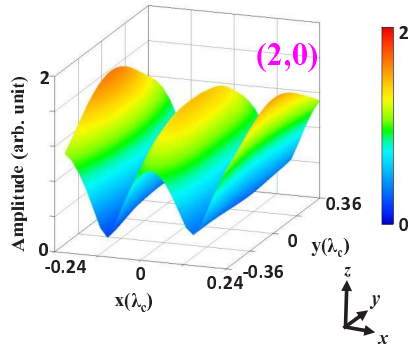

(b)

Figure 4: (color online) The Fourier components of electric fields $E_{z}$ at (a) $V=6.44 V_{p}$, (b) $V=12.7 V_{p}$.

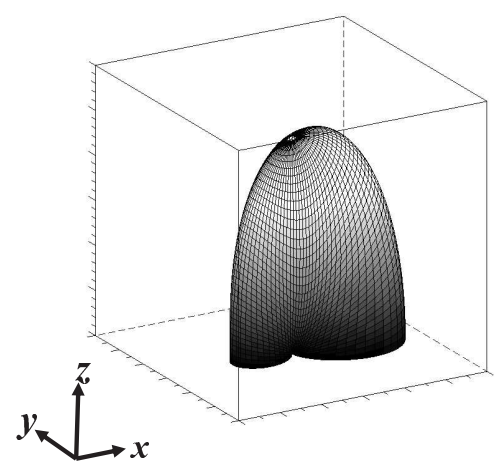

(a)

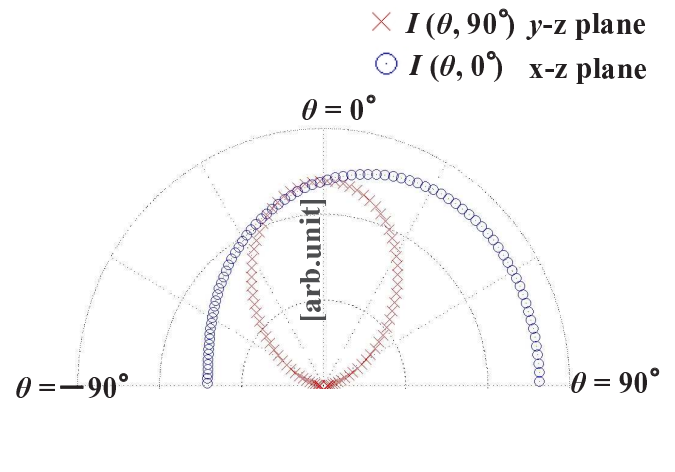

(b)

Figure 5: (color online) (a) The three-dimensional plots of the radiation intensity $I(\theta, \phi)$ at $V=6.44 V_{p}$. (b) The polar plots of $I\left(\theta, 0^{\circ}\right)$ in the $x-z$ plane and $I\left(\theta, 90^{\circ}\right)$ in the $y-z$ plane at $V=6.44 V_{p}$.

reflectional symmetries of the rectangular mesas. Furthermore, the increase of the asymmetric nature of the mesa enhances the mode excitation as can be seen in Fig. 3 (b).

Next, we investigate the internal modes of the mesa at the peak voltages. Hereafter, we show the results for $\gamma=0.2$. To investigate the oscillation part of the electric field, we take the Fourier transform of the electric fields $E_{z}$ in the mesa. Figures 4 (a) and (b) show the amplitude maps of the Fourier components of $E_{z}$ at the $a c$ Josephson frequency for $V=6.44 V_{p}$, and $V=12.7 V_{p}$ respectively. These figures show the appearance of the standing waves corresponding to the cavity resonance modes. However, the amplitudes of the electric fields at the positions of the nodes have finite values. Since the shape of the standing waves are clearly seen in these figures, this fact indicates the existence of "background oscillating modes" which are almost uniform in the mesas.

Furthermore, for clarifying the relation between the internal modes and radiation patterns, we calculate the threedimensional radiation patterns at peak voltages. Figures 5 (a) shows three-dimensional plots of the radiation intensity $I(\theta, \phi)$ at $V=6.44 V_{p}$, and Fig 5 (b) shows the polar plots of $I\left(\theta, 90^{\circ}\right)$ in the $x-z$ plane and $I\left(\theta, 0^{\circ}\right)$ in the $y-z$ plane at $V=6.44 V_{p}$. We can see from these figures that the radiation pattern shows strong asymmetry with respect to the $y-z$ plane and is different from that of the usual patch antenna satisfying the $(1,0)$ cavity resonance condition. This asymmetry is explained by the interference between EM waves emitted by two different radiation sources. As previously mentioned, two type of internal mode, namely, background modes and cavity resonance modes, coexist in the IJJs mesa. Hence, the total EM wave is also described by the sum of the EM waves emitted by these modes. If we assume the perfect uniformity of the background mode, this mode emits the EM wave whose electric field is symmetric with respect to the $y-z$ plane. On the other hand, the $(1,0)$ cavity resonance mode emits the EM wave whose electric field is antisymmetric with respect to the $y-z$ plane. Therefore, radiation intensity becomes strong by constructive interference on one side of the $y-z$ plane and weak by destructive interference on the opposite side 


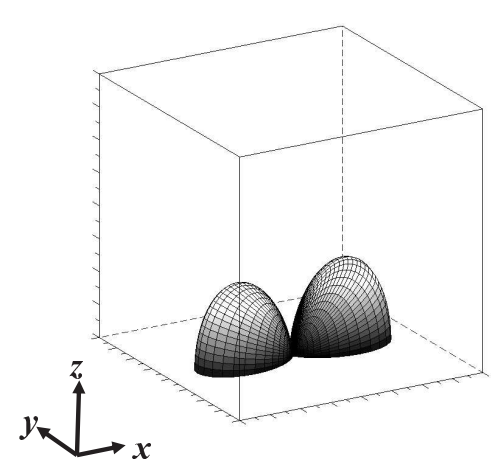

(a)

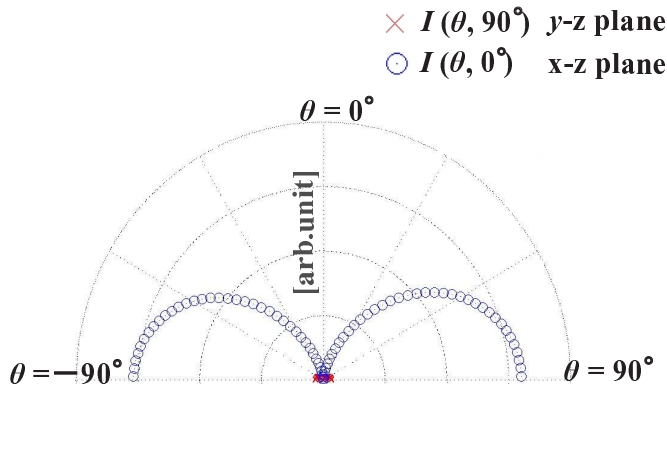

(b)

Figure 6: (color online) (a) The three-dimensional plots of $I(\theta, \phi)$ at $V=12.7 V_{p}$. (b) The polar plots of $I\left(\theta, 0^{\circ}\right)$ in the $x-z$ plane and $I\left(\theta, 90^{\circ}\right)$ in the $y-z$ plane at $V=12.7 V_{p}$.

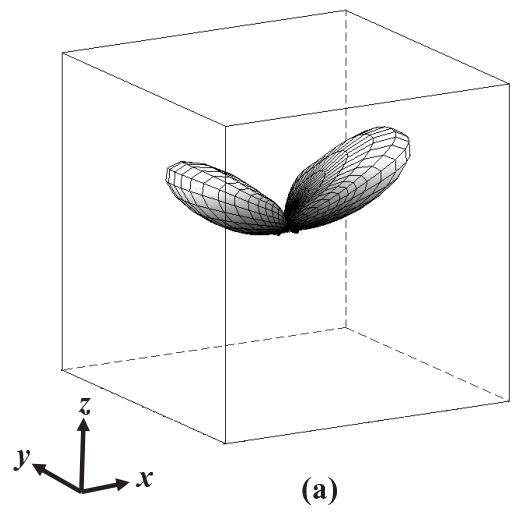

(a)

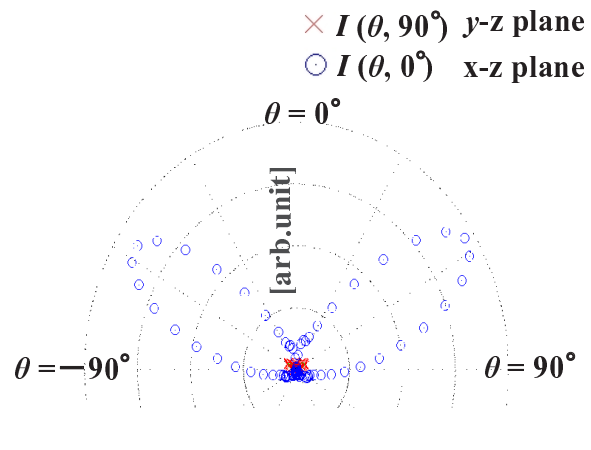

(b)

Figure 7: (color online) The radiation pattern at $V=12.7 V_{p}$ in the presence of finite size substrate. (a) The three-dimensional plots of $I(\theta, \phi)$, and (b) The polar plots of $I\left(\theta, 0^{\circ}\right)$ in the $x-z$ plane and $I\left(\theta, 90^{\circ}\right)$ in the $y-z$ plane.

because of the different symmetries of the radiation waves. In Figs. 6(a) and 6(b), we show $I(\theta, \phi), I\left(\theta, 90^{\circ}\right)$, and $I\left(\theta, 0^{\circ}\right)$ at $V=12.7 V_{p}$, which satisfies the $(2,0)$ cavity resonance condition. The radiation intensity decreases to zero at $\theta=0^{\circ}$, similar to that arising from dipole antenna radiation. In contrast to the result obtained from the $(1,0)$ cavity resonance condition, the radiation pattern is almost symmetric with respect to the $y-z$ plane when the voltage satisfy the $(2,0)$ cavity resonance condition. This is because the $(2,0)$ cavity mode emits the EM wave whose electric field is symmetric with respect to the $y-z$ plane similar to the uniform background mode. In this case, interference between the EM waves emitted by both modes results in similar radiation intensities on each side of the $y-z$ plane. The small asymmetric nature shown in Figs. 6 (a) and (b) reflects the fact that the background mode is slightly asymmetric because of the $j_{c}$ modulation at the hot spot.

Finally we calculate the radiation pattern from the mesa placed on finite size substrate. In the above, we show the radiation patterns from the mesa placed on the infinite size substrate. However, the substrate has finite size in the real system, and the wave diffraction at substrate edges can affect the radiation patterns. The dimensions of the substrate are as follows: width $L_{x}=5.76 \lambda_{c}$, length $L_{y}=5.76 \lambda_{c}$, thickness $L_{z}=0.2 \lambda_{c}$. These sizes of the substrate are similar to those in the experimantal study by Kashiwagi et al. [6]. In Figs. 7(a) and 7(b), we show $I(\theta, \phi), I\left(\theta, 90^{\circ}\right)$, and $I\left(\theta, 0^{\circ}\right)$ at $V=12.7 V_{p}$ with finite size substrate. We can see that these radiation patterns are greatly different from those with infinite size substrate shown in Figs. 6. The interference between the emission wave from the mesa and the diffraction wave from the substrate edges leads to a significant change in radiation patterns. Furthermore, the radiation peaks 
around \pm 60 degrees shown in Figs. 7 were also reported in Kashiwagi's study [6]. These results indicate that the effect of the diffraction at substrate edges are not negligible in the analyses of the experimental radiation patterns.

\section{Conclusion}

In this study, we have studied numerically the three-dimensional radiation patterns from mesa-structured intrinsic Josephson junctions (IJJs). We calculated the electromagnetic fields both inside and outside of IJJs within a inphase approximation. We considered the influence of hot spots in the mesa where $j_{c}$ locally decreases, We observed strong radiation when the ac Josephson frequency satisfies the cavity resonance condition. Due to the existence of the hotspot, the cavity modes in the mesa are strongly excited. For this strong radiation from the IJJs mesa, we calculate the radiation patterns. The radiation patterns reflect the coexistence of two types of internal modes, that is, a uniform background mode and a cavity resonance mode. In particular, the radiation patterns for odd-numbered cavity resonance conditions exhibit asymmetry because of the interference between these two types of radiation waves. Finally, we showed that the radiation patterns were strongly affected by the diffraction at substrate edges. These results indicate that the careful analysis including the diffraction waves is needed for the discussion of the experimental radiation patterns and the internal modes of the mesa.

\section{Acknowledgements}

We wish to thank Dr. S. Kawabata (AIST), Dr. T. Kashiwagi, Dr. H. Minami and Mr. M. Tsujimoto (University of Tsukuba) for valuable discussions and comments. This work was supported in part by CREST-JST (Japan Science and Technology Agency) and Grant-in-Aid for JSPS Fellows.

\section{References}

[1] L. Ozyuzer, A. E. Koshelev, C. Kurter, N. Gopalsami, Q. Li, M. Tachiki, K. Kadowaki, T. Yamamoto, H. Minami, H. Yamaguchi, T. Tachiki, K. E. Gray, W. -K. Kwok, U. Welp Science 318, 1291 (2007)

[2] H. Minami, I. Kakeya, H. Yamaguchi, T. Yamamoto and K. Kadowaki, Appl. Phys. Lett 95, 232511 (2009)

[3] K. Kadowaki, M. Tsujimoto, K. Yamaki, T. Yamamoto, T. Kashiwagi, H. Minami, M. Tachiki, and R. Klemm: J. Phys. Soc. Jpn. 79, 023703 (2010)

[4] M. Tsujimoto, K. Yamaki, K. Deguchi, T. Yamamoto, T. Kashiwagi, H. Minami, M. Tachiki, K. Kadowaki and R. A. Klemm, Phys. Rev. Lett. 105, $037005(2010)$

[5] H. Minami, N. Orita, T. Koike, T. Yamamoto and K. Kadowaki, Physica C 470, S822 (2010)

[6] T. Kashiwagi, K. Yamaki, M. Tsujimoto, K. Deguchi, N. Orita, T. Koike, R. Nakayama, H. Minami, T. Yamamoto, R. A. Klemm, M. Tachiki and K. Kadowaki, J. Phys. Soc. Jpn. 80, 094709 (2011)

[7] T. M. Benseman, A. E. Koshelev, K. E. Gray, W.-K. Kwok, U. Welp, K. Kadowaki, M. Tachiki, and T. Yamamoto, Phys. Rev. B, 84, 064523 (2011)

[8] T. Kashiwagi, M. Tsujimoto, T. Yamamoto, H. Minami, K. Yamaki, K. Delfanazari, K. Deguchi, N. Orita, T. Koike, R. Nakayama, T. Kitamura, M. Sawamura, S. Hagino, K. Ishida, K. Ivanovic, H. Asai, M. Tachiki, R. A. Klemm and K. Kadowaki, Jpn. J. Appl. Phys 51, 010113 (2012).

[9] S. Lin and X. Hu, Phys. Rev. Lett. 100, 247006 (2008)

[10] X. Hu and S. Lin, Phys. Rev. B 78, 134510 (2008)

[11] A. E. Koshelev and L. N. Bulaevskii, Phys. Rev. B 77, 014530 (2008)

[12] A. E. Koshelev, Phys. Rev. B 78, 174509 (2008)

[13] H. Matsumoto, T. Koyama and M. Machida, Physica C 469, 1600 (2009)

[14] T. Koyama,H. Matsumoto, M. Machida and K. Kadowaki, Phys. Rev. B 79, 104522 (2009)

[15] T. Koyama, H. Matsumoto, M. Machida and Y. Ota, Supercond. Sci. Technol. 24, 085007 (2011)

[16] M. Tachiki, S. Fukuya and T. Koyama, Phys. Rev. Lett 102, 127002 (2009)

[17] H. Asai, M. Tachiki, and K. Kadowaki, Phys. Rev. B 85, 064521 (2012).

[18] R. A. Klemm and K. Kadowaki, J. Supercond. Novel Magn. 23, 613 (2010)

[19] R. A. Klemm and K. Kadowaki, J. Phys. Condens. Matter 22, 375701 (2010)

[20] R. A. Klemm, E. R. LaBerge, D. R. Morley, T. Kashiwagi, M. Tsujimoto and K. Kadowaki, J. Phys. Condens. Matter 23, 025701 (2011)

[21] H. B. Wang, S. Guenon, J. Yuan, A. Iishi, S. Arisawa, T. Hatano, T. Yamashita, D. Koelle, and R. Kleiner, Phys. Rev. Lett. 102, 017006 (2009)

[22] H. B. Wang, S. Guenon, B. Gross, J. Yuan, Z. G. Jiang, Y. Y. Zhong, M. Gruenzweig, A. Iishi, P. H. Wu, T. Hatano, D. Koelle, and R. Kleiner, Phys. Rev. Lett. 105, 057002 (2010)

[23] S. Guenon, M. Grunzweig, B. Gross, J. Yuan, Z. G. Jiang, Y. Y. Zhong, M. Y. Li, A. Iishi, P. H. Wu, T. Hatano, R. G. Mints, E. Goldobin, D. Koelle, H. B. Wang, and R. Kleiner, Phys. Rev. B 82, 214506 (2010)

[24] R. J. Luebbers, K. S. Kunz, M. Schneider and F. Hunsberger, IEEE Trans. Antennas Propag. 39, 429 (1991) 